\title{
A PROOF OF A FORMULA IN FOURIER ANALYSIS ON THE SPHERE
}

\author{
JIN-GEN YANG
}

ABSTRACT. A short and elementary proof of a useful formula in spherical harmonic analysis is provided.

In [3] Sherman proved an integral formula for eigenfunctions of the Laplacian on the sphere $S^{n}$. He developed a certain theory of Fourier analysis on the basis of this formula. The purpose of this note is to give an elementary short proof of Sherman's formula.

Let $a=(0, \ldots, 0,1) \in S^{n}, B=\left\{\left(x_{1}, \ldots, x_{n+1}\right) \in S^{n} \mid x_{n+1}=0\right\} . a$ is the "north pole" and $B$ is the "equator". For any integer $k \geqslant 0$ and $b \in B$, define

$$
e_{b, k}(s)=(a+i b, s)^{k}, \quad s \in S^{n},
$$

and

$$
f_{b, k}(s)=\operatorname{sgn}(s, a)^{n-1}(a+i b, s)^{-k-n+1}, \quad s \in S^{n}-B,
$$

where $(\cdot, \cdot)$ is the Euclidean inner product, $i=\sqrt{-1}$. Let $d b$ be the normalized Euclidean measure on $B$.

Theorem (Sherman, Lemma 3.9 of [3]).

$$
\int_{B} e_{b, k}(s) f_{b, k}\left(s^{\prime}\right) d b=P_{k}\left(\left(s, s^{\prime}\right)\right)
$$

for all $s \in S^{n}, s^{\prime} \in S^{n}-B$ and $k>0$, where $P_{k}$ is a polynomial of degree $k$ with $P_{k}(1)=1$, called the (normalized) Gegenbauer polynomial.

Formula (1) corresponds to formula (1.9) in [3].

Proof. Denote the left-hand side of (1) by $F\left(s, s^{\prime}\right)$. Since $F\left(-s,-s^{\prime}\right)=F\left(s, s^{\prime}\right)$ we may assume $\operatorname{sgn}\left(s^{\prime}, a\right)=1$. Let $u_{\phi}$ be the rotation represented by

$$
\left[\begin{array}{cccc}
1 & & & \\
& \ddots & & \\
& & \cos \phi & -\sin \phi \\
& & \sin \phi & \cos \phi
\end{array}\right] .
$$

If $F\left(u_{\phi} s, u_{\phi} s^{\prime}\right)=F\left(s, s^{\prime}\right)$ for all $\phi$ such that $\left(u_{\phi} s^{\prime}, a\right)>0$, then the proof of (1) will be reduced to the case $s^{\prime}=a$ (in which (1) is the standard integral formula for the

Received by the editors October 25, 1982.

1980 Mathematics Subject Classification. Primary 33A45, 33A50; Secondary 26A06.

(C)1983 American Mathematical Society $0002-9939 / 82 / 0000-1273 / \$ 01.50$ 
Gegenbauer polynomial, cf. Theorem 7 of [2] or Lemma 4.2 of [3]) because we can always find some rotation $u$ of $B$ and some $u_{\phi}$ such that $u_{\phi} u s^{\prime}=a$, and it is obvious that $F\left(u s, u s^{\prime}\right)=F\left(s, s^{\prime}\right)$. Hence it is enough to prove $\partial F\left(u_{\phi} s, u_{\phi} s^{\prime}\right) / \partial \phi=0$.

Any point $b \in B$ can be written in the form

$$
b=\left(c_{1} \cos \theta, c_{2} \cos \theta, \ldots, c_{n-1} \cos \theta, \sin \theta, 0\right)
$$

where $c_{1}^{2}+\cdots+c_{n-1}^{2}=1$. Let

$$
\begin{aligned}
& g(c, \theta, \phi, s) \\
& \quad=i \cos \theta \sum_{j=1}^{n-1} c_{j} s_{j}+i \sin \theta\left(s_{n} \cos \phi-s_{n+1} \sin \phi\right)+\left(s_{n} \sin \phi+s_{n+1} \cos \phi\right) .
\end{aligned}
$$

That means $e_{b, k}\left(u_{\phi} s\right)=[g(c, \theta, \phi, s)]^{k}$ and $f_{b, k}\left(u_{\phi} s^{\prime}\right)=\left[g\left(c, \theta, \phi, s^{\prime}\right)\right]^{-k-n+1}$. Then for all integers $m$,

$$
\begin{aligned}
& \frac{\partial\left(g^{m}\right)}{\partial \theta}=m g^{m-1}\left[-i \sin \theta \sum_{j=1}^{n-1} c_{j} s_{j}+i \cos \theta\left(s_{n} \cos \phi-s_{n+1} \sin \phi\right)\right] . \\
& \frac{\partial\left(g^{m}\right)}{\partial \phi}=m g^{m-1}\left[i \sin \theta\left(-s_{n} \sin \phi-s_{n+1} \cos \phi\right)+\left(s_{n} \cos \phi-s_{n+1} \sin \right)\right] .
\end{aligned}
$$

Hence we obtain a useful relation

$$
i \frac{\partial\left(g^{m}\right)}{\partial \phi}-\cos \theta \frac{\partial\left(g^{m}\right)}{\partial \theta}=m g^{m} \sin \theta .
$$

Therefore

$$
\begin{gathered}
\frac{\partial F\left(u_{\phi} s, u_{\phi} s^{\prime}\right)}{\partial \phi}=\frac{\partial}{\partial \phi} \int_{B}[g(c, \theta, \phi, s)]^{k}\left[g\left(c, \theta, \phi, s^{\prime}\right)\right]^{-k-n+1} d b \\
=A \int_{c \in S^{n-2}} d c \int_{-\pi / 2}^{\pi / 2}\left[\frac{\partial\left(g(c, \theta, \phi, s)^{k}\right)}{\partial \phi} g\left(c, \theta, \phi, s^{\prime}\right)^{-k-n+1}\right. \\
\left.+g(c, \theta, \phi, s)^{k} \frac{\partial\left(g\left(c, \theta, \phi, s^{\prime}\right)^{-k-n+1}\right)}{\partial \phi}\right] \cos ^{n-2} \theta d \theta \\
=A \int_{c \in S^{n-2}} d c \int_{-\pi / 2}^{\pi / 2}-i\left[\left(\cos \theta \frac{\partial\left(g(c, \theta, \phi, s)^{k}\right)}{\partial \theta}+k \sin \theta g(c, \theta, \phi, s)^{k}\right)\right. \\
\times\left(g\left(c, \theta, \phi, s^{\prime}\right)^{-k-n+1}+g(c, \theta, \phi, s)^{k}\right. \\
\times\left(\cos \theta \frac{\partial\left(g\left(c, \theta, \phi, s^{\prime}\right)^{-k-n+1}\right)}{\partial \theta}\right.
\end{gathered}
$$


by using (2). Here $d c$ is the ordinary Euclidean measure on $S^{n-2}, A$ is constant. We can easily see that the integrand is $\partial Q / \partial \theta$, where

$$
Q=-i\left[g(c, \theta, \phi, s)^{k} g\left(c, \theta, \phi, s^{\prime}\right)^{-k-n+1} \cos ^{n-1} \theta\right] .
$$

Therefore the integral is zero.

Acknowledgement. The author would like to thank Professor Sigurdur Helgason for correcting some mistakes and giving valuable advice.

\section{REFERENCES}

1. S. Helgason, Topics in harmonic analysis on homogeneous spaces, Birkhauser, Boston, Mass., 1981.

2. C. Müller, Spherical harmonics, Lecture Notes in Math., vol. 17, Springer-Verlag, Berlin and New York, 1966.

3. T. O. Sherman, Fourier analysis on the sphere, Trans. Amer. Math. Soc. 209 (1975), 1-31.

Department of Mathematics, Massachusetts institute of Technology, Cambridge, MasSACHUSETTS 02139 